\title{
CARDINAL FUNCTIONS OF SPACES WITH ORTHO-BASES
}

\author{
By \\ Masami SAKAI
}

\section{§ 1. Introduction.}

Throughout this paper, "space" will mean $T_{1}$-space. Let $\mathscr{B}$ be a base of a space $X . \quad \mathscr{B}$ is said to be an ortho-base if for every $\mathscr{B}^{\prime} \subset \mathscr{B}, \cap \mathscr{B}^{\prime}$ is open or $\mathscr{B}^{\prime}$ is a neighborhood base of some point. $\mathscr{B}$ is said to have subinfinite rank if for every $\mathscr{B}^{\prime} \subset \mathscr{B}$ such that $\cap \mathscr{B}^{\prime} \neq \phi$ and $\mathscr{B}^{\prime}$ is infinite, at least two elements of $\mathscr{B}^{\prime}$ are related by set inclusion. Spaces having an ortho-base, and spaces having a base of subinfinite rank were introduced by Nyikos as natural generalizations of non-archimedean spaces [4] [5].

Concerning cardinal functions of spaces with special bases, Gruenhage showed that for each regular space $X$ having a base of subinfinite rank, $d(X)=h d(X) \geq h l(X)$ $=s(X)$ holds [3], $d(X)$ is the density of $X, h d(X)$ is the hereditary density, $h l(X)$ is the hereditary Lindelöf degree, and $s(X)$ is the spread (i.e., the supremum of the discrete subspaces of $X)$. In this paper we investigate cardinal functions of spaces having ortho-bases. We shall show that $h d(X) \geq h l(X)=s(X)$ holds for each space $X$ having an ortho-base.

\section{§ 2. Main result.}

We need two lemmas. For convenience, for a cardinal $\tau$, we say a space $X$ to be $\tau$-developable if there exist $\tau$ open covers $\left\{\mathcal{H}_{\alpha}\right\}_{\alpha<\tau}$ such that for each $x \in X$ $\left\{\operatorname{St}\left(x, \mathscr{H}_{\alpha}\right)\right\}_{\alpha<\tau}$ is a neighborhood base of $x$.

LeMma 1. Let $X$ be a space having an ortho-base $\mathcal{B}$ and $D$ be the set of isolated points of $X$. If $D$ is dence in $X$, then $X$ is $|D|$-developable.

Proof. Set $D=\left\{d_{\alpha} \mid \alpha<\tau\right\}$, where $\tau$ is a cardinal. For each $x \in X-D$ and $\alpha<\tau$, we take $B_{\alpha}(x) \in \mathscr{B}$ such that $x \in B_{\alpha}(x)$ and $d_{\alpha} \notin B_{\alpha}(x)$. Put $\mathcal{H}_{\alpha}=\left\{\left\{d_{\alpha}\right\} \mid \alpha<\tau\right\} \cup\left\{B_{\alpha}(x) \mid\right.$ $x \in X-D\} . \mathscr{H}_{\alpha}$ is obviously an open cover of $X$. Let $x$ be a point of $X$ and $W$ be a neighborhood of $x$. If $x \in D$, then $\operatorname{St}\left(x, \mathcal{H}_{\alpha}\right)=\{x\} \subset W$ for some $\alpha$. So, we assume $x \in X-D$. Suppose that St $\left(x, \mathcal{H}_{\alpha}\right) \oplus W$ for any $\alpha<\tau$. Then for each $\alpha$, we can take $H_{\alpha} \in \mathcal{H}_{\alpha}$ such that $x \in H_{\alpha}$ and $H_{\alpha} \notin W$. Since $\left\{H_{\alpha}\right\}_{\alpha<\tau}$ can not be a neigh- 
borhood base of $x, H=\bigcap_{\alpha<\tau} H_{\alpha}$ must be open. But $H \cap D=\phi$, because $H_{\alpha} \nsupseteq d_{\alpha}$. Since $D$ is dense in $X$, this is a contradiction.

The following lemma is well known in the countable case and can be easily carried over to the general case. So we omit the proof.

Lemma 2. Let $X$ be $\tau$-developable. If the cardinality of each closed discrete subspace is at most $\tau$, then $X$ is $\tau$-Lindelof (i.e., every open cover has a subcover of the cardinality $\tau$ ).

THEOREM 3. Let $X$ be a space having an ortho-base $\mathscr{B}$. Then $h d(X) \geq s(X)=$ $h l(X)$ holds.

Proof. Since $h d(X) \geq s(X)$ and $h l(X) \geq s(X)$ are obvious, we show $s(X) \geq h l(X)$. Let $s(X)=\tau$. Since for each subspace $Y$ of $X, s(Y) \leq \tau$ and $Y$ has an ortho-base, the proof is complete if we show that $X$ is $\tau$-Lindelöf. Suppose that there exists an open cover $U$ of $X$ which has not a subcover of the cardinality $\tau$. Firstly we take $x_{0} \in X$ and $U_{0} \in \mathcal{U}$ such that $x_{0} \in U_{0}$. Put $V_{0}=U_{0}$. Let $\gamma<\tau^{+}$. We assume that for each $\beta<\gamma$ we could take $x_{\beta} \in X$ and an open set $V_{\beta}$ such that the following (*) is satisfied.

(*) $\left\{\begin{array}{l}V_{\beta} \cap\left\{x_{\alpha} \mid \alpha<\gamma\right\}=\left\{x_{\beta}\right\} \quad \text { for each } \beta<\gamma . \\ \text { There exists } U_{\beta} \in \mathcal{U} \text { such that } V_{\beta} \subset U_{\beta} \text { for each } \beta<\gamma .\end{array}\right.$

Then, if we set $A=\left\{x_{\alpha} \mid \alpha<\gamma\right\}$, since $|A| \leq \tau, \mathrm{Cl} A$ is $\tau$-Lindelöf by Lemma 1 and Lemma 2, Thus $\mathrm{Cl} A \cup\left(\underset{\beta<\gamma}{\cup} V_{\beta}\right)$ is covered by $\tau$ elements of $U$. So we can take $x_{r} \in X-\mathrm{Cl} A \cup\left(\bigcup_{\beta<\gamma} V_{\beta}\right)$. We take $U_{r} \in \mathcal{U}$ and an open set $V_{r}$ such that $x_{r} \in V_{r} \subset U_{r}$ and $V_{r} \cap A=\phi$. Now by the induction we get the discrete space $\left\{x_{\alpha} \mid \alpha<\tau^{+}\right\}$. This is a contradiction to $s(X)=\tau$.

There exists a space having an ortho-base such that $h d(X) \neq d(X)$. In fact the space in $[6,3.6 . I]$ is such a space.

Concerning SH (Souslin's hypothesis), we note the following theorem.

THEOREM 4. The following (a), (b) and (c) are equivalent.

(a) $\mathrm{SH}$ is false.

(b) There exists a non-metrizable non-archimedean space such that $s(X)$ is countable.

(c) There exists a non-metrizable regular space having an ortho-base such that $s(X)$ is countable. 
Proof. The equivalence of (a) and (b) is due to [1]. Also, refer [5, Theorem 1.7]. (b) $\rightarrow$ (c) is trivial. We show (c) $\rightarrow($ b). Let $X$ be a space of (c). Since by Theorem $3 X$ is regular Lindelöf, it is paracompact. Therefore $X$ is a protometrizable space (i.e., paracompact space having an ortho-base). It follows from Fuller's result [2, Theorem 6$]$ that $X$ is the perfect irreducible image of a nonarchimedean space $Y$. Since metrizability is an invariant of perfect maps, $Y$ is not metrizable. Since the spread of a non-archimedean space is equal to the cellularity, by the irreducibility of the map, $s(Y)$ must be countable. Thus $Y$ is the desired space.

COROllaRy 5. The following $(a)$ and $(b)$ are equivalent.

(a) SH.

(b) Each regular space having an ortho-base is metrizable if the spread is countable.

\section{References}

[1] Arhangel'skii, A.V. and Filippov, V.V., Spaces with bases of finite rank. Math. USSR Sbornik, 16 (1972), 147-158.

[2] Fuller, L. B., Trees and proto-metrizable spaces. Pacific J. Math. 104 (1983), 55-75.

[3] Gruenhage, G., Some results on spaces having an ortho-base or a base of subinfinite rank. Topology Proceed. 2 (1977), 151-159.

[4] Lindgren, W.F. and Nyikos, P. J., Spaces with bases satisfying certain order and intersection properties. Pacific J. Math. 66 (1976), 455-476.

[5] Nyikos, P. J., Some surprising base properties in topology. Studies in Topology, Academic Press. (1975), 427-450.

[6] Engelking, R., General Topology, Warszawa 1977.

Institute of Mathematics

University of Tsukuba

Ibaraki, 305 Japan 